\title{
Schizophrenia as a progressive brain disorder
} Rene Kahn*

Address: Department of Psychiatry, Rudolf Magnus Institute of Neuroscience, University Medical Center Utrecht, The Netherlands

* Corresponding author

from International Society on Brain and Behaviour: 2nd International Congress on Brain and Behaviour Thessaloniki, Greece. 17-20 November 2005

Published: 28 February 2006

Annals of General Psychiatry 2006, 5(Suppl I):S6 doi:10.1186/I744-859X-5-SI-S6

Brain imaging studies have consistently demonstrated brain abnormalities in patients with schizophrenia. These changes are largely confined to decreases in gray matter volumes and enlargement of the lateral and third ventricles. To date schizophrenia has been considered to result from abnormalities in neurodevelopment, with brain changes to be static. However, schizophrenia has long been thought to be a progressive or a degenerative, not a developmental, disorder. Indeed, Kraepelin considered the progressive clinical deterioration to be the hallmark of the disorder, naming it dementia praecox to reflect this particular aspect. Lately, others have re-emphasised the importance of the decline in functioning in schizophrenia as a clue to its pathogenesis, suggesting that the brain abnormalities in schizophrenia could be expected to reflect this clinical progression. Indeed, we and others have reported brain abnormalities to increase over time in schizophrenia. Interestingly, not all patients show changes in brain volumes over time: we demonstrated that the changes are particularly pronounced in those patients with a poor prognosis in the first years of illness. Moreover progressive changes are most pronounced in the frontal and temporal areas as postulated by Kraepelin over a hundred years ago. Interestingly, white matter did not change over time. This fits with a recent finding from our group in $\mathrm{MZ}$ and $\mathrm{DZ}$ twins discordant for schizophrenia showing that white matter changes appear related to the risk of developing schizophrenia and gray matter changes tom be related to the disease. 